\title{
miR-329 inhibits papillary thyroid cancer progression via direct targeting WNT1
}

\author{
LIANG WU ${ }^{1}$, FULAI PEI $^{1}$, XIAOJUAN MEN $^{2}$, KAI WANG $^{2}$ and DELIANG MA ${ }^{1}$ \\ ${ }^{1}$ Department of Oncology, Linyi Central Hospital, Linyi, Shandong 276400; \\ ${ }^{2}$ Department of Breast Surgery, Weifang People's Hospital, Weifang, Shandong 261041, P.R. China
}

Received January 13, 2018; Accepted June 29, 2018

DOI: $10.3892 /$ ol.2018.9102

\begin{abstract}
Dysregulated microRNA-329 (miR-329) serves an important role in the progression of certain types of tumor. However, the exact function and mechanisms of miR-329 in papillary thyroid cancer (PTC) remain unknown. The present study investigated the function and mechanisms of miR-329 in regulating PTC cell progression. The results revealed that the expression of miR-329 was significantly downregulated in PTC tissues and cell lines compared with adjacent normal tissues and a human immortalized follicular cell line. miR-329 mimics notably decreased PTC cell proliferation, colony formation and WNT1 expression in vitro, as well as suppressing PTC tumor growth in vivo. In addition, luciferase assays determined that miR-329 was able to directly bind with the 3'untranslated region of WNT1. Furthermore, short interfering RNA-WNT1-induced downregulation of WNT1, which demonstrated similar effects to miR-329 overexpression. WNT1 overexpression rescued the tumor suppressive effects of miR-329 in PTC cells. The present study provided new insights into the role of miR-329 in PTC progression and suggests the potential application of miR-329 as a therapy for PTC.
\end{abstract}

\section{Introduction}

Thyroid cancer is the most common tumor of the endocrine system, and its incidence rate has remarkably increased over the past several decades (1). Thyroid cancer can be classified into four types depending on pathological type: Papillary, follicular, medullary and anaplastic thyroid cancer. Papillary thyroid cancer (PTC) is the most common type of thyroid malignant tumor that accounts for approximately $90 \%$ of all thyroid cancer (2). In most cases, patients with PTC have an excellent prognosis after they undergo surgical resection

Correspondence to: Dr Deliang Ma, Department of Oncology, Linyi Central Hospital, 17 Jian Kang Road, Yishui, Linyi, Shandong 276400, P.R. China

E-mail: madeliang2018@163.com

Key words: papillary thyroid cancer, migration and invasion, proliferation, microRNA-329, WNT1 combined with radioiodine and levothyroxine treatment. However, $10-15 \%$ of patients suffering from PTC with relapses and distant metastases frequently elicit a poor response to standard treatments and achieve poor clinical outcomes (3). Therefore, molecular mechanisms underlying the formation and progression of PTC must be elucidated to improve its diagnosis, therapy and prevention.

MicroRNAs (miRNAs) are a group of small non-coding RNAs that regulate gene expression through translation repression or mRNA degradation by binding to the 3'-untranslated region of a target mRNA (4). miRNAs are involved in the regulation of cell survival, proliferation and migration by mediating the expression of their target genes (5). Alterations in miRNA expression are likely implicated in PTC development and progression. miR-329 located on 14q32.31 participates in the progression of several cancers (6-13). However, the expression levels, biological functions and associated molecular mechanism of miR-329 in PTC have yet to be elucidated.

In the present study, we measured miR-329 expression in PTC tissues and cell lines. We also investigated the regulatory roles of miR-329 in PTC cells. Moreover, we explored the underlying molecular mechanism of its actions in PTC cells.

\section{Materials and methods}

Tissue sample collection. Paired PTC and adjacent non-tumor tissues were collected from 20 patients who underwent surgical resection at Linyi Central Hospital. All patients had not received chemotherapy or radiotherapy before surgery. Tissue samples were collected during surgery, frozen in liquid nitrogen, and then stored until total RNAs were extracted. Informed consent was obtained from each patient, and the study protocol and consent procedures were approved by the ethics committee of Linyi Central Hospital (Linyi, China).

Cell culture and cell transfection. Human PTC cell lines (TPC-1 and BCPAP) and human immortalized follicular cell line (Nthy-ori3-1) were obtained from the ATCC (Manassas, VA, USA). BCPAP originally classified as a thyroid gland papillary cancer but is now considered to be a poorly differentiated thyroid gland cancer (14). In the present study, BCPAP acts as poorly differentiated thyroid cancer cell line to further confirm the experiments results 
of TPC-1 and not affect the outcomes of this study. The cell lines were authenticated using short-tandem repeat profiling with BMR Genomics. The cells were cultured in DMEM (HyClone; GE Healthcare Life Sciences, Logan, UT, USA) supplemented with $10 \%$ fetal bovine serum (HyClone; GE Healthcare Life Sciences) in a humidified atmosphere with $5 \% \mathrm{CO}_{2}$ and humidified sphere of $95 \%$ at $37^{\circ} \mathrm{C}$. miR-329 mimic and negative control (miR-NC) were purchased from GE Healthcare Life Sciences. A lentiviral packaging kit was purchased from Shanghai GeneChem (Shanghai, China). Lentiviruses carrying miR-329 or miR-NC were packaged in HEK293T cells and harvested from the culture supernatant in accordance with the kit manufacturer's instructions. Stable cell lines were established by selecting infected TPC-1 and BCPAP cells with puromycin (HyClone; GE Healthcare Life Sciences). WNT1 overexpression plasmid was achieved using pcDNA3.1/WNT1 transfection. siRNA targeting human Wnt1 was obtained from Santa Cruz Biotechnology, Inc., (sc-36839; Dallas, TX, USA). siWnt1 (5 nmol) was transfected into the cells according to the manufacturer's protocol. Transient transfection was performed with a Lipofectamine 2000 Reagent in accordance with the manufacturer's instruction.

Colony formation assay. Cell proliferation was analyzed by using the plate colony formation assay. A total of 400 cells from each group were seeded in a new six-well plate and cultured for approximately 10 days until colony formation was observed. The colonies were fixed with methanol and stained with $1 \%$ crystal violet (Beyotime Institute of Biotechnology, Haimen, China). Images of the colonies were obtained, and the colonies with more than 50 cells were counted under a microscope (Olympus Corporation, Tokyo, Japan).

CCK- 8 assay. The viability of TPC-1 and BCPAP cells was determined by using the CCK- 8 assay. TPC- 1 and BCPAP cells stably transfected with miR-329 mimic or miR-NC were seeded in 96-well plates at a density of $1 \times 10^{3}$ cells per well and then cultured for 24,48 , and $72 \mathrm{~h}$ before performing the CCK-8 assay. After $4 \mathrm{~h}$ of incubation with CCK-8 at $37^{\circ} \mathrm{C}$, the absorbance (OD value) at a wavelength of $450 \mathrm{~nm}$ was detected and used for calculating cell viability.

Cell migration and invasion assays. Cell migration and invasion assays were analyzed by using Transwell chamber in accordance with the manufacturer's instruction. For the invasion assay, the upper sides of the filters were coated with $50 \mu 1$ of Matrigel (BD Biosciences, Franklin Lakes, NJ, USA). TPC-1 and BCPAP cells stably transfected with miR-329 mimic or miR-NC were plated at a density of $5 \times 10^{4}$ cells per well in the upper chamber without serum. The lower chamber was filled with $600 \mu \mathrm{l}$ of the DMEM medium with $10 \% \mathrm{FBS}$ to act as the nutritional attraction. After incubation for $8 \mathrm{~h}$ (migration) and $24 \mathrm{~h}$ (invasion), the cells on the upper membrane surface were removed, whereas the invasive cells attached to the lower surface of the membrane insert were fixed with $70 \%$ methanol for $30 \mathrm{~min}$ and stained with $0.1 \%$ crystal violet for $10 \mathrm{~min}$. Then, the cells were counted in five randomly selected fields per well under a light microscope (Olympus Corporation).
$R N A$ extraction and quantitative real time PCR ( $q P C R)$. Total RNAs (inclusive of miRNAs) were extracted from cells or xenograft tissues using Trizol reagent (Invitrogen; Thermo Fisher Scientific, Inc., Waltham, MA, USA) in accordance with the manufacturer's protocol. To measure the expression levels of miR-329, qPCR assay was performed, and RNU48 served as the internal control. To analyze the mRNA levels of WNT1, total RNAs were reversely transcribed by oligodT primer using PrimeScript RT Reagent kit (Takara Biotechnology Co., Ltd., Dalian, China). GAPDH served as the endogenous control. The relative expression levels of miR-329 and WNT1 were calculated as the inverse log of $\Delta \Delta \mathrm{CT}$ and normalized to the reference. The primers used for amplification were as follows: miR-329 Forward 5'-GGGAACACACCTGGTTAAC-3', Reverse 5'-CAGTGCGTGTCGTGGAGT-3' RNU48 Forward 5'-TGATGATGACCCCAGGTAACTCTGAGTG-3', Reverse 5'-GTCAGAGCGCTGCGGTGATGGCATCAGC-3' GAPDH Forward 5'-GACCTGACCTGCCGTCTAG-3' Reverse 5'-ACT CCTGCTTGCTGATCCAC-3' WNT1 Forward 5'-AGCCCT AGCTGCCAACAGTA-3' Reverse 5'-GGAATTGCCATT TGCACTCT-3'

Western blot analysis. Equal amounts of the protein from lysates of PTC cells were subjected to $10 \%$ SDS-PAGE and then transferred onto PVDF membranes. The immunoreactive bands were first incubated with the primary antibodies, including WNT1 (1:500) and GAPDH (1:1,000; both from Santa Cruz Biotechnology, Inc.) antibodies. The intensity of protein bands was detected by Image-Pro Plus v.6.0 software (National Institutes of Health, Bethesda, MD, USA). GAPDH served as the loading control.

Dual luciferase reporter assay. The wild-type (WT) and mutated putative (mut) miR-329 target sequences in the WNT1 3'UTR were amplified from human WNT1 cDNA by PCR and then cloned into the SacI and HindIII sites of the pmiRNA-report firefly luciferase vector (GeneChem). TPC-1 and BCPAP cells were seeded in a 24-well plate and co-transfected with the WT or MUT reporter plasmid, a Renilla luciferase plasmid, and miR-329 mimic or miR-NC. Luciferase activities were measured $24 \mathrm{~h}$ after transfection using a dual luciferase assay kit (Promega Corporation, Madison, WI, USA). Firefly luciferase activity was normalized to its corresponding Renilla luciferase activity.

Tumourigenesis assay in nude mice. All experimental procedures involving animals were approved by the Ethics Committee of Linyi Central Hospital (Shandong, China). 5-week-old BALB/c athymic nude mice $(n=12$, female, weight range; 20-22 g) (Jilin University, Changchun, China) and maintained in a SPF environment with constant humidity $(45-50 \%)$ and constant temperature $\left(25-27^{\circ} \mathrm{C}\right)$ under a $12 \mathrm{~h}$ light/dark cycle with free access to food and water. BCPAP cells stably transformed with miR-329 or miR-NC were inoculated subcutaneously into the right flanks of nude mice. Each experimental group included six mice. Tumor growth was measured every 7 days from injection using a digital caliper, and the tumor volume was calculated by the following formula: tumor volume $=\left(\right.$ length $\mathrm{x}$ width $\left.{ }^{2}\right) / 2$. After $42 \mathrm{~d}$, the mice were sacrificed, and xenografts were harvested. 
Statistical analysis. Statistical analyses were performed using SPSS v.13.0 software (SPSS, Inc., Chicago, IL, USA). Differences between two groups were assessed using Student's t-test (two-tailed). Data of more than two groups were analyzed using one way ANOVA with post hoc test by Tukey's test. Correlations between WNT1 and miR-329 were analyzed using Spearman rank correlation. Each experiment was performed at least three times. $\mathrm{P}<0.05$ was considered to indicate a statistically significant difference and results are represented as means \pm standard deviations (SD).

\section{Results}

miR-329 is downregulated in PTC tissues and cell lines. The expression levels of miR-329 in 20 paired samples (PTC specimens and corresponding adjacent non-tumor tissues) were detected via qPCR. Results showed that miR-329 expression was remarkably lower in tumor tissues compared to adjacent normal tissues (Fig. 1A). In addition, the expression of miR-329 in PTC cell lines (TPC-1, BCPAP) was significantly reduced compared to that in the human immortal follicular thyroid cell Nthy-ori3-1 (Fig. 1B).

Overexpression of $\mathrm{miR}-329$ reduced the cell proliferation in PTC cells. The overexpression of miR-329 was achieved by transfection with miR-329 mimic in TPC-1 and BCPAP, as verified using qPCR assays (Fig. 2A). As exhibited by CCK-8 assays, the cell viability of TPC-1 and BCPAP cells was significantly inhibited after miR-329 overexpression compared to that of cells transfected with miR-NC (Fig. 2B and C). In addition, results of the colony formation assay illustrated that the proliferation of miR-329 overexpression cells was significantly decreased relative to that of cells transfected with miR-NC (Fig. 2D and E). Furthermore, the results of the western blot revealed that miR-329 overexpression significantly decreased the expression of the WNT1 oncogene (Fig. 2F).

miR-329 inhibited the migration and invasion capability of PTC cells in vitro. To evaluate the migration and invasion potential of PTC cells transfected with miR-329 mimic, trasnswell assay was performed in vitro. The overexpression of miR-329 significantly decreased the number of PTC cells capable of migration and invasion (Fig. 3A-D). These results suggested that miR-329 reduced the migration and invasion of PTC cells in vitro.

WNT1 was a direct target gene of $m i R-329$. We identified WNT1 mRNA as one of the putative miR-329 targets by using the microrna.org and Targetscan 7.2 (http://www. targetscan.org/vert 72/) (Fig. 4A). To demonstrate whether miR-329 affects WNT1 expression in PTC, we examined the expression of WNT1 in TPC-1 and BCPAP cells transfected with miR-329 mimic. The data showed that levels of WNT1 mRNA (Fig. 4B) and protein (Fig. 4C) were both decreased in TPC-1 and BCPAP cell transfected with miR-329 mimic. To further verify that WNT1 mRNA is a direct target of miR-329, luciferase reporter assays was analyzed. The results illustrated that luciferase activity was remarkedly reduced by miR-329 transduction in TPC-1 and BCPAP cells expressing a reporter driven by the WT WNT1 3'-UTR, but not the mutated WNT1
3'-UTR (Fig. 4D and E). Furthermore, we showed that the mRNA expression of miR-329 was reversely correlated with WNT1 in PTC tissues (Fig. 4F).

WNT1 silencing inhibited PTC cell viability, proliferation, migration and invasion in vitro. To further determine whether WNT1 play a critical role in PTC progression, we performed in vitro function loss analyses by WNT1 silencing with siWNT1. Results of western blot showed that the expression of WNT1 was significantly decreased in TPC-1 and BCPAP cells (Fig. 5A). Cell viability was detected via CCK-8 assay. The results showed that knockdown of WNT1 in PTC cells significantly inhibited cell viability (Fig. 5B and C). Furthermore, the colony formation assay showed that the proliferation of WNT1 silencing cells was significantly inhibited (Fig. 5D). In addition, the transwell migration and invasion assay showed that the WNT1 silencing significantly decreased the migration and invasion capability of TPC-1 and BCPAP cells (Fig. 5E and F).

miR-329 suppressed tumor growth in vivo. To evaluate the effects of miR-329 on tumor progression in vivo, we extended our investigation by subcutaneous implantation of stably overexpressing miR-329 BCPAP cells in nude mice. Tumor volume were examined every $7 \mathrm{~d}$ for $42 \mathrm{~d}$, at which point the tumors were removed for photograph (Fig. 6A) and analysis. As shown in Fig. 6B and $\mathrm{C}$, the results showed that the volumes and weight of xenografts derived from cells with miR-329 mimic were significantly smaller than that of miR-NC group. In addition, the qPCR results showed that the expression of miR-329 was significantly enhanced in xenografts from miR-329 mimic-transfected cells (Fig. 6D). However, the mRNA expression of WNT1 was remarkedly decreased in the xenografts tissues derived from the cells transfected with miR-329 mimic (Fig. 6E).

Overexpression of WNT1 overcame the miR-329-mediated inhibition of cell progression. We investigated whether WNT1 overexprssion rescued the progression inhibition effect of miR-329 mimic in PTC cells. The expression of WNT1 was analyzed by western blot (Fig. 2F). Results of CCK-8 (Fig. 2B and C) and colony formation assay (Fig. 2D and E) showed that WNT1 overexprssion reversed the inhibition of cell viability and proliferation. Results of transwell migration and invasion assay showed that WNT1 overexpression significantly reversed the inhibition of cell migration and invasion via regulating WNT1 in PTC cell (Fig. 3A-D).

\section{Discussion}

In the present study, we showed that miR-329 was downregulated in human PTC tissues compared with that in adjacent normal tissues, and its levels were lower in the PTC cell lines TPC1 and BCPAP than in the normal cell line. Further studies illustrated that miR-329 overexpression suppressed PTC cell viability, proliferation, migration and invasion in TPC1 and BCPAP cells. The present study also demonstrated that miR-329 directly targeted WNT1 to inhibit the biological behaviour of PTC cells. Spearman's correlation analysis revealed that the expression of miR-329 was inversely correlated with mRNA expression of WNT1 in PTC tissues. miR-329 also suppressed 

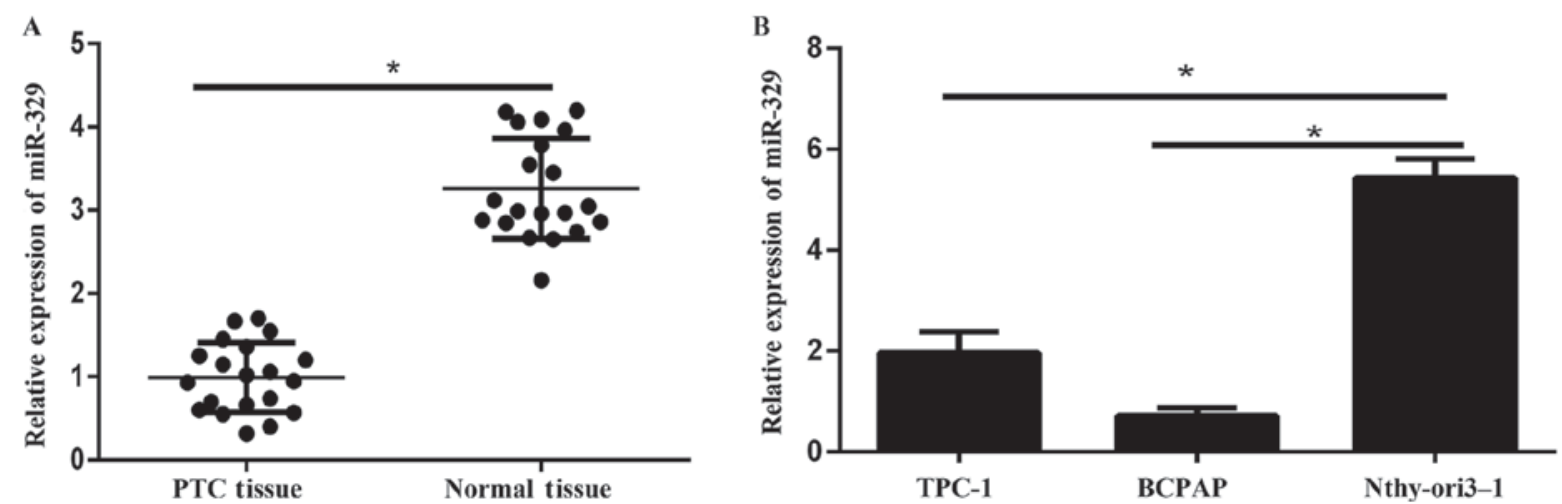

Figure 1. miR-329 is downregulated in PTC tissues and cell lines. (A) Relative miR-329 expression levels were analyzed via quantitative polymerase chain reaction in 20 pairs of PTC tissues and adjacent normal tissues. (B) The expression level of miR-329 was analyzed in PTC cell lines (TPC-1 and BCPAP) and human immortalized follicular cell line (Nthy-ori3-1). RNU48 served as an internal control. "P<0.05. PTC, papillary thyroid cancer.
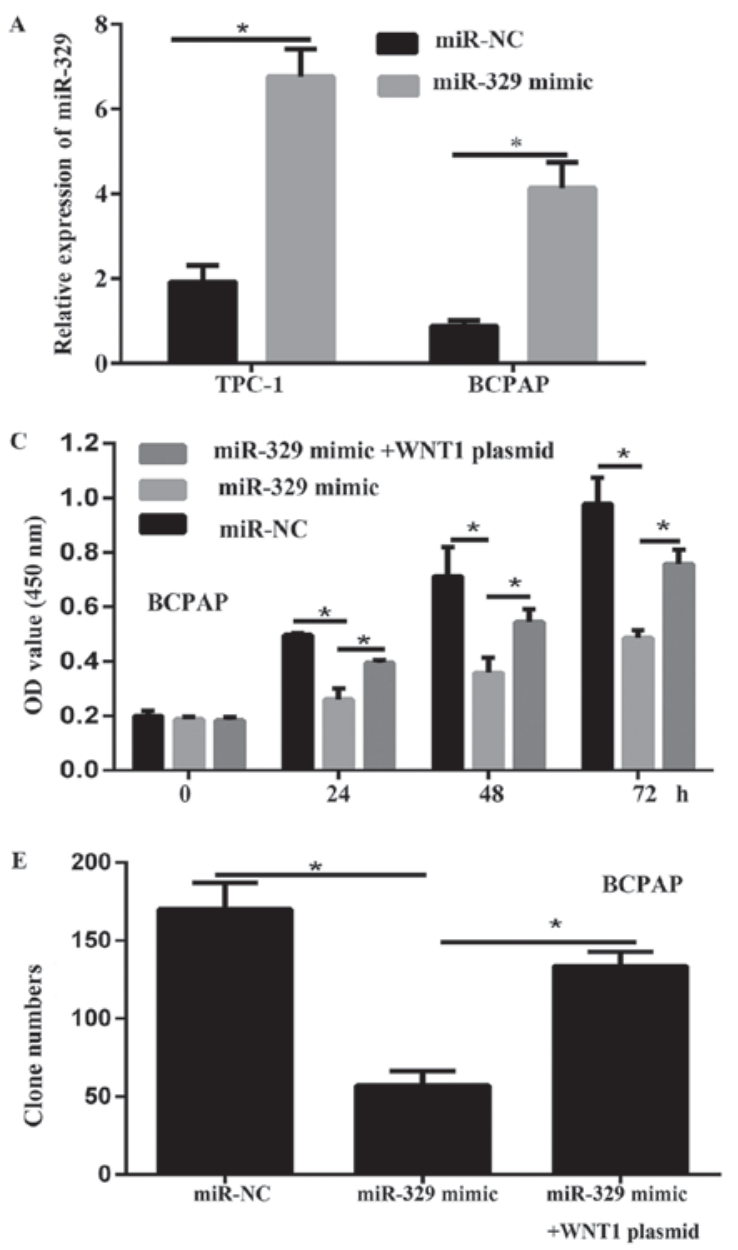

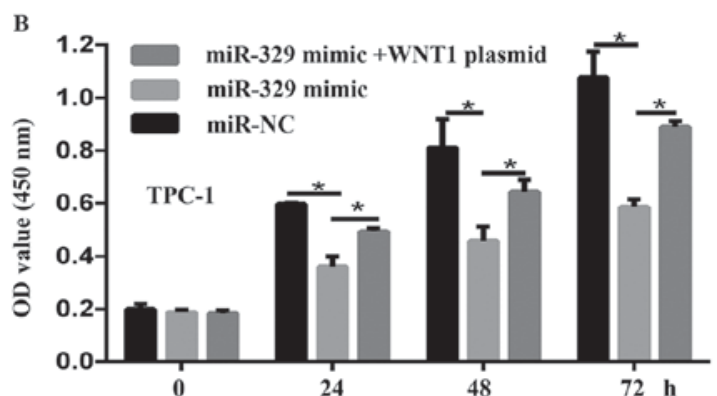

D
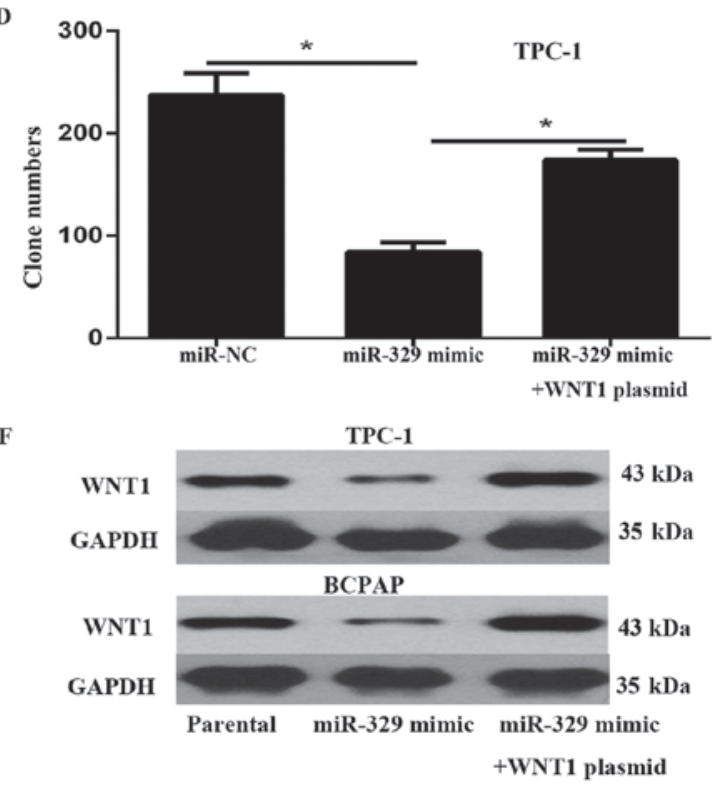

Figure 2. miR-329 decreases proliferation in PTC cells, while the overexpression of WNT1 rescued this effect. (A) TPC-1 and BCPAP cells were transfected with miR-329 mimic or miR-NC and WNT1 plasmid, the expression level of miR-329 was detected via quantitative polymerase chain reaction. RNU48 served as an internal control. The cell viability of (B) TPC-1 and (C) BCPAP cells was determined by a CCK- 8 assay. The proliferation of (D) TPC-1 and (E) BCPAP cells was assessed by colony formation assays. (F) Western blot assays were performed to confirm the WNT1 protein expression in TPC-1 and BCPAP cells ${ }^{*} \mathrm{P}<0.05$. PTC, papillary thyroid cancer; miR, microRNA; NC, negative control.

tumor growth in nude mice by inhibiting WNT1. These findings illustrated that miR-329 inhibited the development and progression of PTC.

Previous studies reported that miR-329 expression is decreased in several different types of cancers (6-13). Wang et al (6), reported that miR-329 was downregulated in pancreatic cancer and involved in the inhibition of pancreatic cancer progression by regulating the GRB2/pERK pathway (6). miR-329 might partially inhibit neuroblastoma progression by targeting KDM1A (7) and play an important role in lung cancer progression through the inhibition of cell proliferation, migration, invasion and apoptosis by targeting 

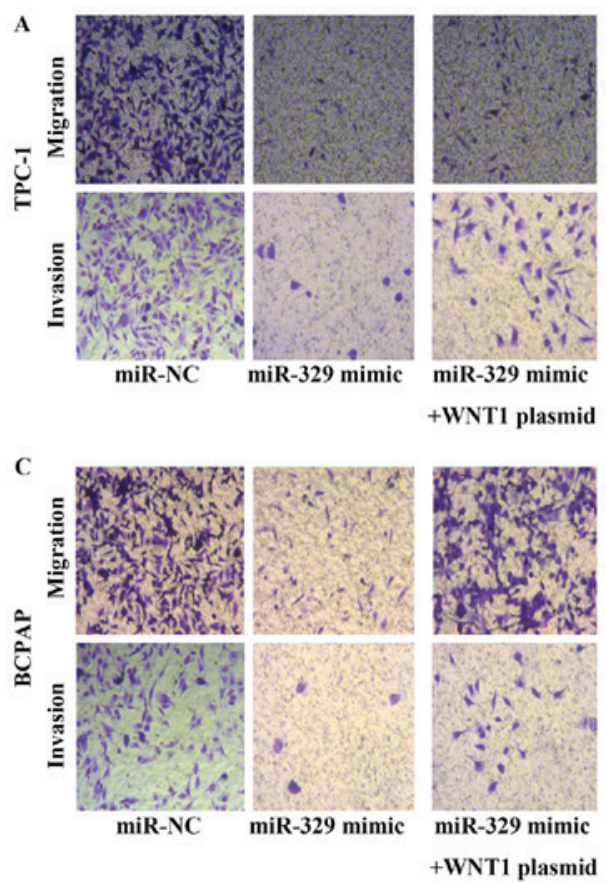
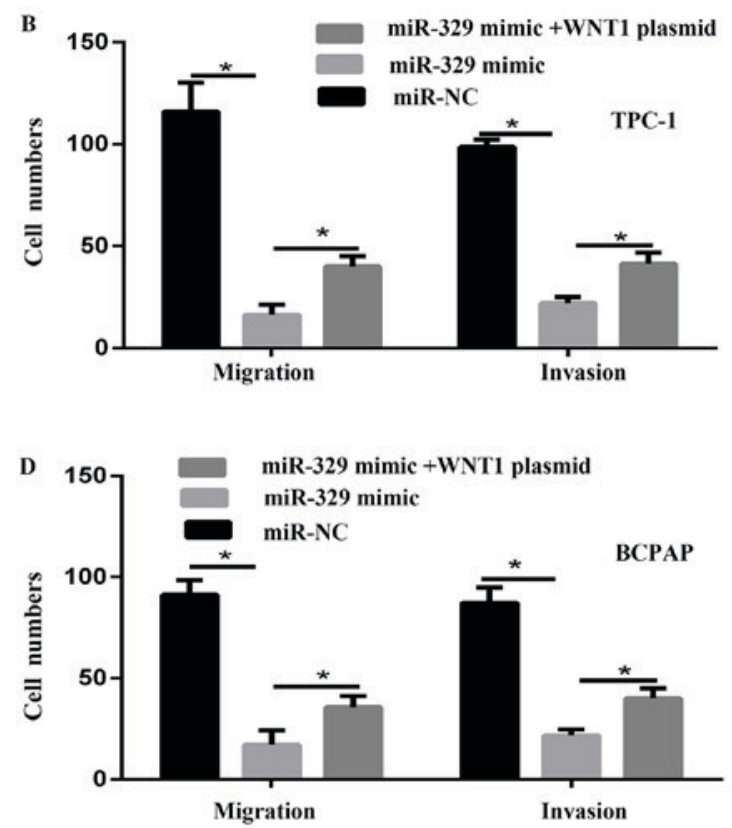

Figure 3. miR-329 overexpression decreases cell migration and invasion in TPC-1 and BCPAP cells, while WNT1 overexpression rescued this effect. (A-D) TPC-1 and BCPAP cells were co-transfected with either miR-329 mimic or miR-NC and WNT1 plasmid. Transwell assays were conducted and quantified. Migrated and invasive cells were fixed, stained and counted and images were captured (magnification, x200). (A) Images and (B) quantification of TPC-1 cell migration and invasion in the lower chamber. (C) Images and (D) quantification of BCPAP cell migration and invasion in the lower chamber. "P<0.05. miR, microRNA; NC, negative control.

A

\section{$5^{\prime}$-ccugccuccucaucaGUGUGUa-3' WT WNT1 \\ 3'- uuucuccaauuggucCACACAa -5' miR-329 \\ $5 '$ - ccugccuccucaucaCACACAa-3' MUT WNT1}

C
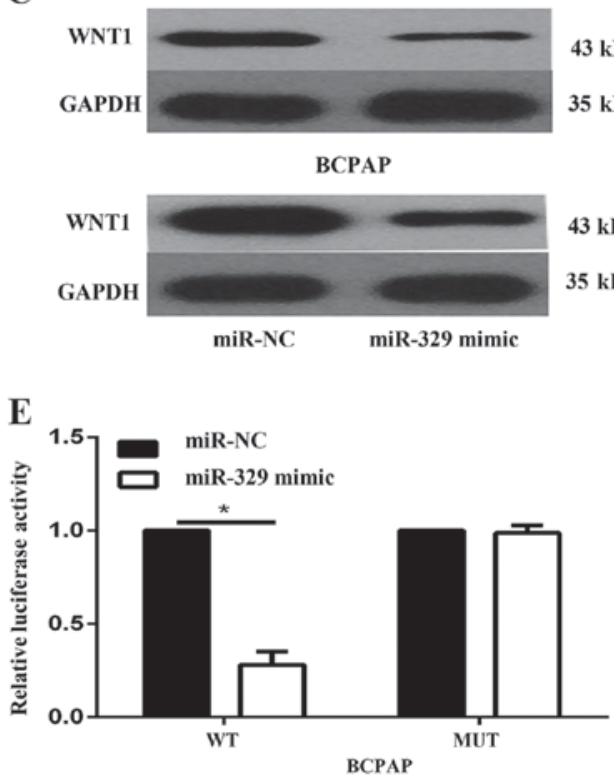

B

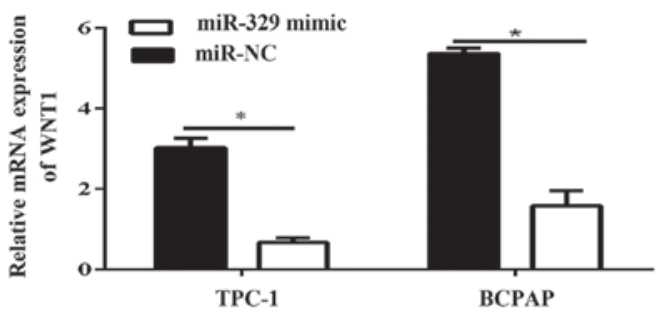

D

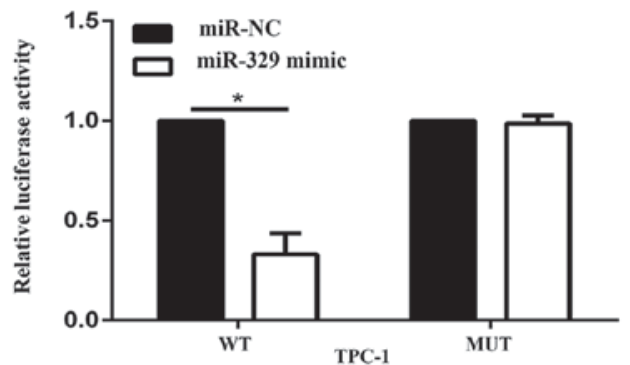

$\mathbf{F}$

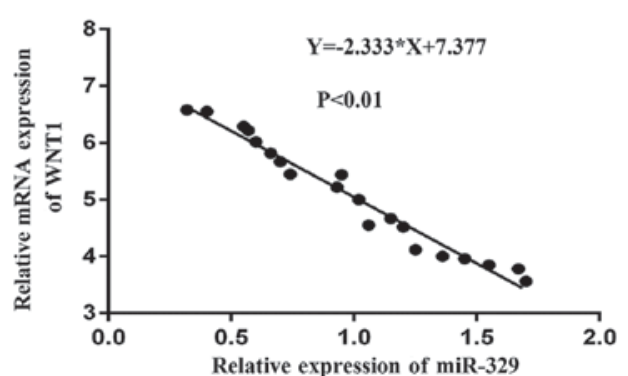

Figure 4. WNT1 is a direct target gene of miR-329. (A) Sequence alignment of miR-329 with 3'untranslated region of WNT1 predicted by TargetScan. (B) The mRNA expression level of WNT1 in TPC-1 and BCPAP cells was analyzed by quantitative polymerase chain reaction. GAPDH served as the control. (C) Western blot analysis was used to analyze the protein expression of WNT1 in TPC-1 and BCPAP cells. GAPDH served as the control. Luciferase activity of (D) TPC-1 and (E) BCPAP cells was examined via luciferase reporter assay. (F) Spearman's correlation analysis examined the correlations between the mRNA expression level of WNT1 and miR-329 in PTC tissues. ${ }^{*} \mathrm{P}<0.05$. miR, microRNA; NC, negative control; WT, wild type; MUT, mutant. 
A

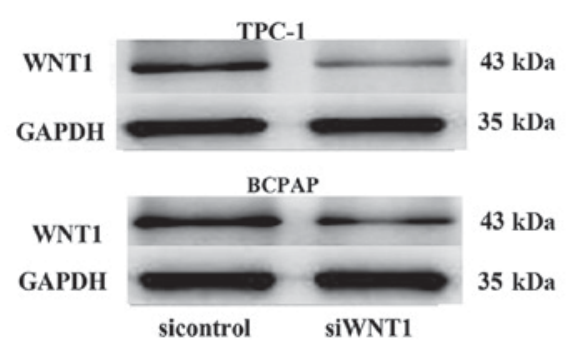

C

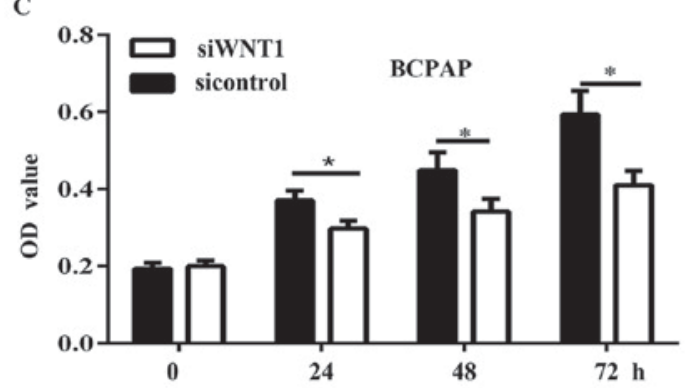

E
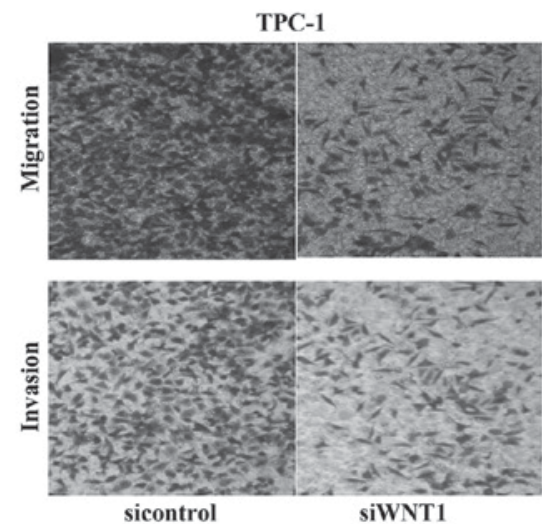

F
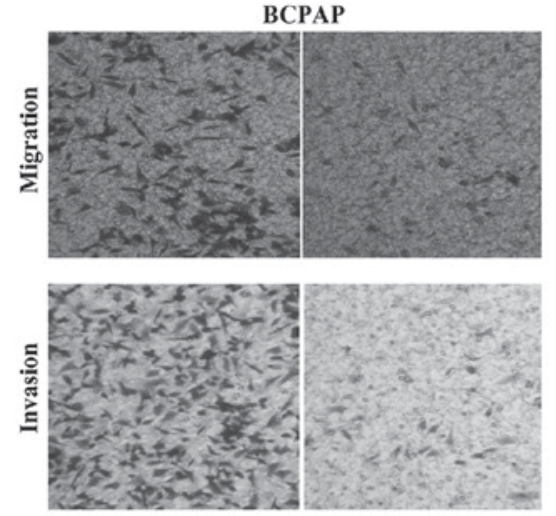

sicontrol
B
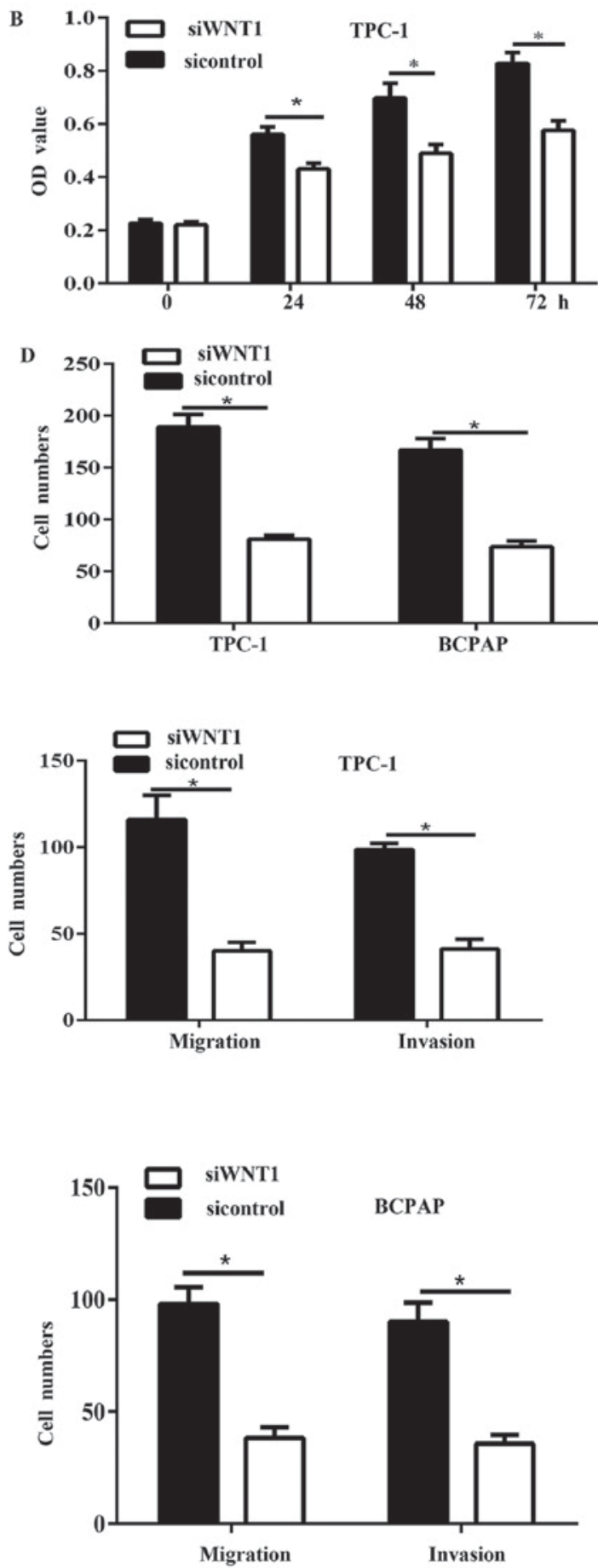

Figure 5. WNT1 silencing inhibits PTC cell viability, proliferation, migration and invasion in vitro. (A) Western blot analysis examined the expression of WNT1 in the TPC-1 and BCPAP cells transfected with siWNT1 or sicontrol. Cell viability in (B) TPC-1 and (C) BCPAP cells was examined via a CCK-8 assay. (D) Proliferation was examined by a colony formation assay. The migration and invasion capability of in (E) TPC-1 and (F) BCPAP cells was determined by Transwell migration and invasion assays. ${ }^{*} \mathrm{P}<0.05$. si, short interfering RNA. PTC, papillary thyroid cancer.

oncogenic MET (9). In gastric cancer progression, miR-329 possibly prevented cell proliferation, migration and invasion by targeting TIAM1 (10). However, expression of miR-329 in oral squamous cell carcinoma specimens was increased compared with that in matched tissue ulcerative colitis, suggesting its function as an oncogene (11). Therefore, miR-329 performed dual functions: tumor suppressor or oncogene dependent on specific cancer types. In the present study, results showed that expression of miR-329 was downregulated in PTC tissues and cell lines, suggesting the tumor suppressive role of miR-329 in PTC progression. To further illustrate the function of miR-329 in PTC development, we conducted cell transfection and found that miR-329 overexpression significantly reduced the viability, proliferation and migration and invasion of PTC cells. These results illustrated that miR-329 repressed tumour progression in PTC carcinogenesis, and further demonstrated that 
A

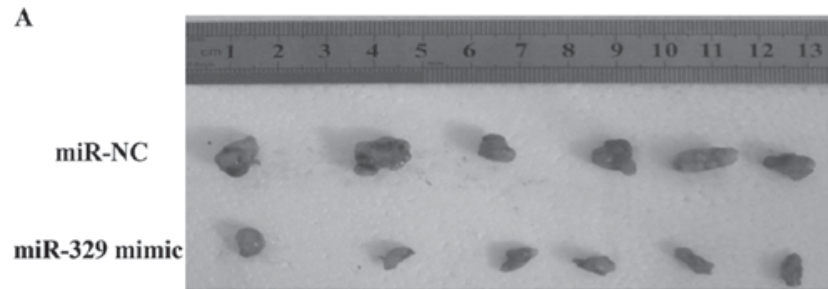

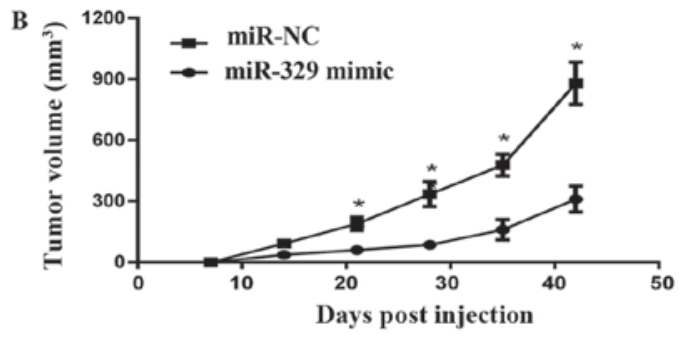

C

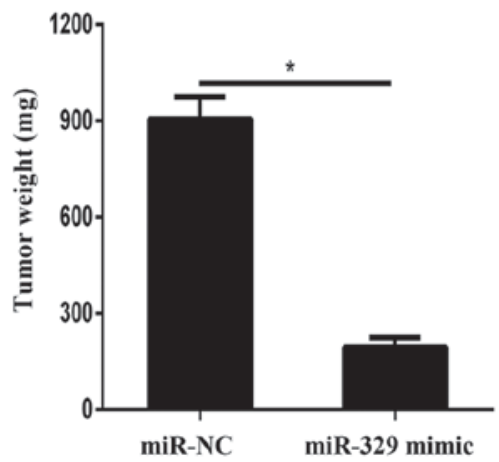

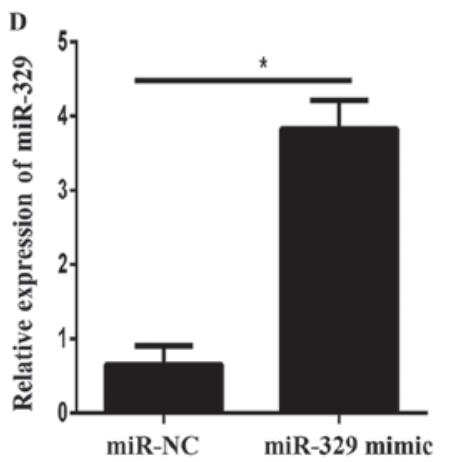

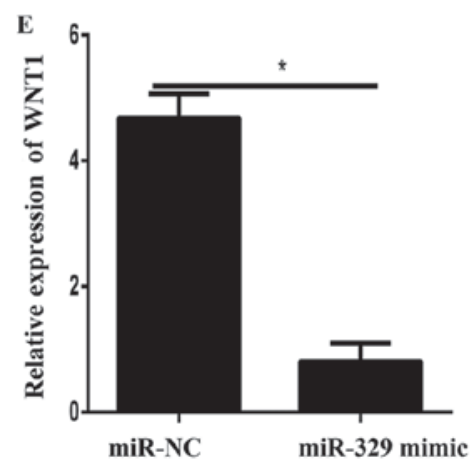

Figure 6. miR-329 inhibits tumor growth in vivo via downregulation of WNT1. (A) Images of tumors derived from the BCPAP-miR-329 and BCPAP-miR-NC groups. (B) Tumor volume was analyzed every 7 days for 42 days following injection. (C) Tumor weight in BCPAP-miR-329 and BCPAP-miR-NC groups. (D) qPCR was used to determine the (D) miR-329 and (E) WNT1 expression in each treatment group. ${ }^{\text {P }}<0.05$. miR, microRNA; NC, negative control; qPCR, quantitative polymerase chain reaction.

the same gene might serve as oncogene or tumor suppressor depending on the type of tissue and the context in which they were expressed.

Many miR-329 targets have been identified in various types of cancer. In the present study, WNT1 was determined as a target gene of miR-329 in PTC. miR-329 could also regulate the viability, proliferation and metastasis of PTC cells by targeting WNT1. WNT1, the first member of the 19 known members of the human Wnt family, has been shown to promote cancer progression because it triggers cell proliferation and metastasis $(15,16)$. WNT1 binds to specific Frizzled surface receptors of cells to activate different signalling pathways, resulting in the accumulation and nuclear localisation of the downstream molecule $\beta$-catenin protein (17-19). In this study, an important molecular association between miR-329 and WNT1 was demonstrated. Dual luciferase reporter assays illustrated that WNT1 was one of the direct target genes of miR-329. Furthermore, the ectopic expression of WNT1 remarkably enhanced the viability, proliferation and migration and invasion of PTC cells reduced by miR-329 mimic, suggested that WNT1 might be one of the functional target genes of miR-329. In addition, the mRNA expression of WNT1 was inversely correlated with miR-329 levels in PTC tissues. At last, the knockdown of WNT1 repressed PTC cell viability, proliferation and migration and invasion in vitro. These results implied WNT1 is a functional target gene of miR-329 in PTC.

In summary, the present study is the first to provide evidence that miR-329 is downregulated in thyroid cancer tissues and cell lines. miR-329 also functions as a tumor suppressor of PTC growth by targeting WNT1. This newly identified miR-329/WNT1 link provides new insight into the mechanisms underlying PTC development, and suggests that targeting the miR-329/WNT1 axis may represent a promising therapeutic strategy for PTC treatment. Nevertheless, further studies are needed to determine the exact mechanism of decreased miR-329 expression during the progression of PTC and to further explore other possible targets of miR-329 in PTC.

\section{Acknowledgements}

Not applicable.

\section{Funding}

No funding was received.

\section{Availability of data and materials}

All data generated or analyzed during this study are included in this published article.

\section{Authors' contributions}

LW and DM conceived and designed the experiments. DM wrote and revised the manuscript. FP, XM and KW conducted the experiments. All authors read and approved the final manuscript.

\section{Ethics approval and consent to participate}

The present study was approved by the Ethics Committee of Linyi Central Hospital. All patients provided written informed consent.

\section{Patient consent for publication}

All patients provided written informed consent for the publication of their data and any accompanying images. 


\section{Competing interests}

The authors declare that they have no competing interests.

\section{References}

1. Liebner DA and Shah MH: Thyroid cancer: Pathogenesis and targeted therapy. Ther Adv Endocrinol Metab 2: 173-195, 2011.

2. Gonzalez-Gonzalez R, Bologna-Molina R, Carreon-Burciaga RG, Gómezpalacio-Gastelum M, Molina-Frechero $\mathrm{N}$ and Salazar-Rodriguez S: Papillary thyroid carcinoma: Differential diagnosis and prognostic values of its different variants: Review of the literature. ISRN Oncol 2011: 915925, 2011.

3. Shi X, Liu R, Basolo F, Giannini R, Shen X, Teng D, Guan H, Shan Z1, Teng W, Musholt TJ, et al: Differential clinicopathological risk and prognosis of major papillary thyroid cancer variants. J Clin Endocrinol Metab 101: 264-274, 2016.

4. Valinezhad Orang A,Safaralizadeh R and Kazemzadeh-Bavili M: Mechanisms of miRNA-mediated gene regulation from common downregulation to mRNA-specific upregulation. Int J Genomics 2014: 970607, 2014

5. Macfarlane LA and Murphy PR: MicroRNA: Biogenesis, function and role in cancer. Curr Genomics 11: 537-561, 2010.

6. Wang X, Lu X, Zhang T, Wen C, Shi M, Tang X, Chen H, Peng C, Li H, Fang Y, et al: mir-329 restricts tumor growth by targeting grb2 in pancreatic cancer. Oncotarget 7: 21441-21453, 2016.

7. Yang H, Li Q, Zhao W, Yuan D, Zhao H and Zhou Y: miR-329 suppresses the growth and motility of neuroblastoma by targeting KDM1A. FEBS Lett 588: 192-197, 2014.

8. Wang P, Luo Y, Duan H, Xing S, Zhang J, Lu D, Feng J, Yang D, Song L and Yan X: MicroRNA 329 suppresses angiogenesis by targeting CD146. Mol Cell Biol 33: 3689-3699, 2013.

9. Sun CC, Li SJ, Zhang F, Pan JY, Wang L, Yang CL, Xi YY and Li de J: Hsa-miR-329 exerts tumor suppressor function through down-regulation of MET in non-small cell lung cancer. Oncotarget 7: 21510-21526, 2016.

10. Li Z, Yu X, Wang Y, Shen J, Wu WK, Liang J and Feng F: By downregulating TIAM1 expression, microRNA-329 suppresses gastric cancer invasion and growth. Oncotarget 6: 17559-17569, 2015.
11. Shiah SG, Hsiao JR, Chang WM, Chen YW, Jin YT, Wong TY, Huang JS, Tsai ST, Hsu YM and Chou ST: Downregulated miR329 and miR410 promote the proliferation and invasion of oral squamous cell carcinoma by targeting Wnt-7b. Cancer Res 74: 7560-7572, 2014.

12. Xiao B, Tan L, He B, Liu Z and Xu R: miRNA-329 targeting E2F1 inhibits cell proliferation in glioma cells. J Transl Med 11: 172,2013

13. Li W, Liang J, Zhang Z, Lou H, Zhao L, Xu Y and Ou R: MicroRNA-329-3p targets MAPK1 to suppress cell proliferation, migration and invasion in cervical cancer. Oncol Rep 37: 2743-2750, 2017.

14. Saiselet M, Floor S, Tarabichi M, Dom Hébrant A, van Staveren WC and Maenhaut C: Thyroid cancer cell lines: An overview. Front Endocrinol (Lausanne) 3: 133, 2012.

15. Benad P, Rauner M, Rachner TD and Hofbauer LC: The anti-progestin RU-486 inhibits viability of MCF-7 breast cancer cells by suppressing WNT1. Cancer letters 312: 101-108, 2011.

16. Stanczak A, Stec R, Bodnar L, Olszewski W, Cichowicz M, Kozlowski W, Szczylik C, Pietrucha T, Wieczorek M and Lamparska-Przybysz M: Prognostic significance of Wnt-1, $\beta$-catenin and E-cadherin expression in advanced colorectal carcinoma. Pathol Oncol Res 17: 955-963, 2011.

17. Wieczorek M, Paczkowska A, Guzenda P, Majorek M, Bednarek AK and Lamparska-Przybysz M: Silencing of Wnt-1 by siRNA induces apoptosis of MCF-7 human breast cancer cells. Cancer Biol Ther 7: 268-274, 2008.

18. Wang JM, Huang FC, Kuo MH, Wang ZF, Tseng TY, Chang LC, Yen SJ, Chang TC and Lin JJ: Inhibition of cancer cell migration and invasion through suppressing the Wnt1-mediating signal pathway by G-quadruplex structure stabilizers. J Biol Chem 289: 14612-14623, 2014.

19. Sasaya K, Sudo H, Maeda G, Kawashiri S and Imai K: Concomitant loss of p120-catenin and $\beta$-catenin membrane expression and oral carcinoma progression with E-cadherin reduction. PLoS One 8: e69777, 2013.

(i) $\Theta$ This work is licensed under a Creative Common EY NG ND Attribution-NonCommercial-NoDerivatives 4.0 International (CC BY-NC-ND 4.0) License. 